\title{
OPEN Morphological and vascular characteristics of the optic nerve head of normal guinea pigs
}

\author{
Lei Guo ${ }^{1,2,6}$, Jun Tao ${ }^{3,6}$, Yang Tong ${ }^{2}$, Shichao Chen ${ }^{2}$, Xin Zhao ${ }^{2}$ \& Rui Hua ${ }^{4,5} \llbracket$
}

The morphological and vascular characteristics of the optic nerve head (ONH) of normal guinea pigs have not been fully recognized. Therefore, we aimed to investigate them using optical coherence tomography (OCT) and optical coherence tomography angiography (OCTA). We measured the refractive error, axial length, and intraocular pressure (IOP) and performed OCT and OCTA of the ONH of 3- and 4-week-old tricolour guinea pigs. A total of 208 right eyes from 208 normal guinea pigs were examined. The refractive error (both myopic and hyperopic) of the 3-week group was significantly higher than that of the 4-week group $(p<0.001)$, the IOP of the 3-week group was significantly lower than that of the 4-week group ( $p=0.014)$, and the circumpapillary retinal nerve fibre layer (cpRNFL) of the 3-week group was significantly thicker than that of the 4-week group $(p=0.048)$. There were no significant differences in the average vessel area, vascular density, total number of junctions, total vessel length, total number of endpoints, and vascular diameter between the two groups. However, an age-adjusted linear regression analysis revealed that the total vessel length was positively associated with the cpRNFL thickness $(p=0.024)$ and negatively associated with IOP $(p=0.016)$. This is the first report on morphological and vascular characteristics of the $\mathrm{ONH}$ in normal guinea pigs based on in vivo OCT and OCTA imaging and quantification of ONH parameters. These results may contribute to further research on myopia using guinea pig models.

The optic nerve head $(\mathrm{ONH})$ is an important structure involved in the pathogenesis of myopia ${ }^{1}$. Guinea pigs have been increasingly used as experimental models of myopia ${ }^{2}$. In an in vitro study, Ostrin et al. reported the structural anatomy of the retina and $\mathrm{ONH}$ in guinea pigs ${ }^{3}$. Haematoxylin and eosin-stained sections showed retinal ganglion cell axons organised into fascicles in the prelaminar and laminar regions of the $\mathrm{ONH}$, and scanning electron microscopy revealed a well-defined lamina cribrosa with radially oriented collagen beams ${ }^{3}$.

Optical coherence tomography (OCT) and OCT angiography (OCTA) are recent technological advancements for the diagnosis, treatment, and follow-up of several retinal diseases due to their ability to capture highresolution characteristics of the retina within seconds ${ }^{4}$. In our previous study, we found that a U-shaped neural canal and a lower ratio of Bruch membrane opening to minimum rim width were early indicators of retinal nerve fibre layer (RNFL) defects in pre-perimetric glaucomatous eyes of glaucoma patients with normal circumpapillary RNFL (cpRNFL) thickness in OCT images 5 . Jnawali et al. observed that histological sections of all the retinal layers in guinea pigs were consistent with their OCT images, and hyper-reflective regions of the OCT images corresponded to the retinal pigment epithelium, inner/outer segment junction, outer plexiform layer, inner plexiform layer, and $\mathrm{RNFL}^{2}$. However, the anterior lamina cribrosa surface cannot be visualised in OCT images because the thick RNFL and vascular sheath obscure the ONH, which decreases the penetrance of the scanning laser. The reliability of OCTA for measuring choroidal thickness and choroidal blood perfusion has been verified in guinea pigs $^{6}$. In addition, OCTA-related parameters-including vascular density, number of vessel junctions, total vessel length, and number of vessel endpoints-have been introduced to assess choroidal neovascularisation in rat models; these parameters showed significantly higher values for the intermediate and deep vascular plexi of choroidal neovascularisation when obtained by OCTA than when obtained by fluorescein angiography ${ }^{7}$. Similar to the $\mathrm{ONH}$ in humans, the $\mathrm{ONH}$ of guinea pigs also contains vascular tissue. Protruding vascular tufts in the

\footnotetext{
${ }^{1}$ Ophthalmology and Optometry Centre, The First Hospital of China Medical University, Shenyang, China. ${ }^{2}$ Department of Ophthalmology, Shenyang Xingqi Eye Hospital, Shenyang, China. ${ }^{3}$ Department of Ophthalmology, The 4th People's Hospital of Shenyang, Shenyang, China. ${ }^{4}$ Department of Ophthalmology, The First Hospital of China Medical University, No. 155, Nanjingbei Street, Heping District, Shenyang 110001, Liaoning Province, China. ${ }^{5}$ Macular Disease Research Laboratory, Department of Ophthalmology, West China Hospital, Sichuan University, Chengdu, China. ${ }^{6}$ These authors contributed equally: Lei Guo and Jun Tao. ${ }^{\square}$ email: woodshua@126.com
} 
ONH have been reported in guinea pigs ${ }^{2}$; however, little is known about the normal imaging characteristics of their blood perfusion and OCTA parameters. Quantification of such parameters in normal guinea pig models is critical for characterising vascular structural changes expected to occur in conditions like myopia.

In the present study, we aimed to investigate and set the normal ranges for both morphological and blood perfusion characteristics of the ONH in normal guinea pigs using OCT and OCTA and investigate the factors influencing blood perfusion in the $\mathrm{ONH}$. We conducted this study to provide normative data for guinea pigs considered potentially valuable models for myopia.

\section{Methods}

Animals. Our study was conducted on 3- and 4-week-old tricolour guinea pigs stratified by their age. Threefour week guinea pigs are successful models for myopia ${ }^{6}$. Because there are no statistically significant differences between the refractive and biometric measurements (intraocular pressure [IOP] and axial length $[\mathrm{AL}]$ ) in the right and left eyes ${ }^{2,8}$, only the right eye was selected for the study in all groups. Guinea pigs with congenital cataracts, corneal abnormalities, or other ocular diseases that may affect vision were excluded at the baseline. All animal care and examinations in this study conformed to the ARVO Statement for the Use of Animals in Ophthalmic and Vision Research, and all experimental protocols were approved by the Animal Care and Use Committee of China Medical University. The study is reported according to the ARRIVE guidelines (https:// arriveguidelines.org).

Parameter measurements. Initially, IOP was measured in all eyes using a tonometer (Icare Finland Oy, Vantaa, Finland). The IOPs of the guinea pigs have been demonstrated to undergo diurnal variations, peaking in the early morning and decreasing across the day $^{3}$; therefore, measurements were always taken at the same time of day (afternoon) to prevent confounding effects of diurnal rhythms. Three sets of six measurements were recorded, and the average IOPs were calculated. Guinea pigs do not require anaesthesia for IOP measurements; therefore, anaesthesia-induced alterations in IOP can be prevented ${ }^{2}$.

And then, topical tropicamide eye drops were administered three times at an interval of 5 min for cycloplegia, and assessments were performed $30 \mathrm{~min}$ later. Cycloplegic refractive error was measured using a streak retinoscope (YZ-24, Suzhou Liuliu, China).

The ONH was assessed using spectral-domain OCT (Cirrus HD-OCT, Zeiss, Germany) in the ONH mode. We designed the examining pallet for animal studies, and the focus plane of OCT was adjusted for imaging of the $\mathrm{ONH}$ without an animal customized cornea lens for OCT examination. The positions of the eyes of the guinea pigs were controlled and fixed by regulating the stem fixed in the mouth. All the OCT parameters originated from the instrument's software except for calculating the parameters of OCTA, which were achieved by AngioTool software11 (a custom program outside of the Cirrus). The analysed parameters were average cup-to-disc ratio, cpRNFL thickness ( $\mu \mathrm{m}$, the RNFL of the circle with a diameter of $3.4 \mathrm{~mm}$ centred at the disc), and cup volume $\left(\mathrm{mm}^{3}\right)$. Blood flow signals of the ONH were also recorded on OCTA (Cirrus HD-OCT, Zeiss, Germany) within the $6 \times 6-\mathrm{mm}$ range. SD-OCT and OCTA measurements were performed under general anaesthesia (intraperitoneal anaesthesia) with $1 \%$ pentobarbital sodium $(0.33 \mathrm{ml} / 100 \mathrm{~g})$.

Finally, when the pupil returned to normal, AL was measured using an A-scan ultrasound (Aviso, Quantel Medical Inc., Cournon-d'Auvergne, France; probe diameter, $5 \mathrm{~mm}$; ultrasonic resolution, $0.01 \mathrm{~mm}$; ultrasonic transmission frequency, $11 \mathrm{~Hz}$ ) under topical anaesthesia.

Analysis of OCTA images. Guinea pig eyes do not have any retinal vessels outside the ONH region, which is proven by fundus angiography Phoenix-OCT fluorescence angiography in our previous unpublished study (Glaucomatous abnormalities following scleral cross-linking in form-deprivation myopic guinea pig eyes [under consideration]). In this study, the blood flow signal maps of ONH were obtained using the OCTA device, and the retinal avascular layer mode was selected. The retinal avascular layer mode of the Cirrus OCT is used for automatic segmentation of the retinal vasculature. However, no automatic segmentation mode for the $\mathrm{ONH}$ is available for OCTA, and we used this retinal avascular layer mode to analyse the vasculature of the ONH based on our observation. Subsequently, these target images were imported to the AngioTool software ${ }^{9}$, which automatically measured the vessel area, vascular density, total number of junctions, total vessel length, and total number of endpoints of vessels of the $\mathrm{ONH}$ (Fig. 1) ${ }^{10}$. The vascular diameter was defined as the vessel area divided by the total vessel length. We consider that the nutrient vessels within the $\mathrm{ONH}$, which was showed by OCTA, may be central retinal vessels and their capillary system. However, no related histopathological studies have been reported until now. Nevertheless, we believe that this vascular system was responsible for the nutrition of the optic nerve, which may play an important role in the progression of myopia.

Statistical analyses. All data were analysed using SPSS 24.0 statistical software (IBM, SPSS Statistics, Version 24, SPSS Inc., Chicago, USA). The data are expressed as mean \pm standard deviation. Independent samples t-test was used for pair-wise comparisons of AL, IOP, refraction, and OCT- and OCTA-related parameters for the 3- and 4-week old groups. Pearson's test was used to determine the correlations between vascular density, total vessel length in the $\mathrm{ONH}$, and all other parameters. The factors influencing vascular density and total vessel length of the $\mathrm{ONH}$ were determined using linear regression analysis. A p-value of $<0.05$ was considered statistically significant. 

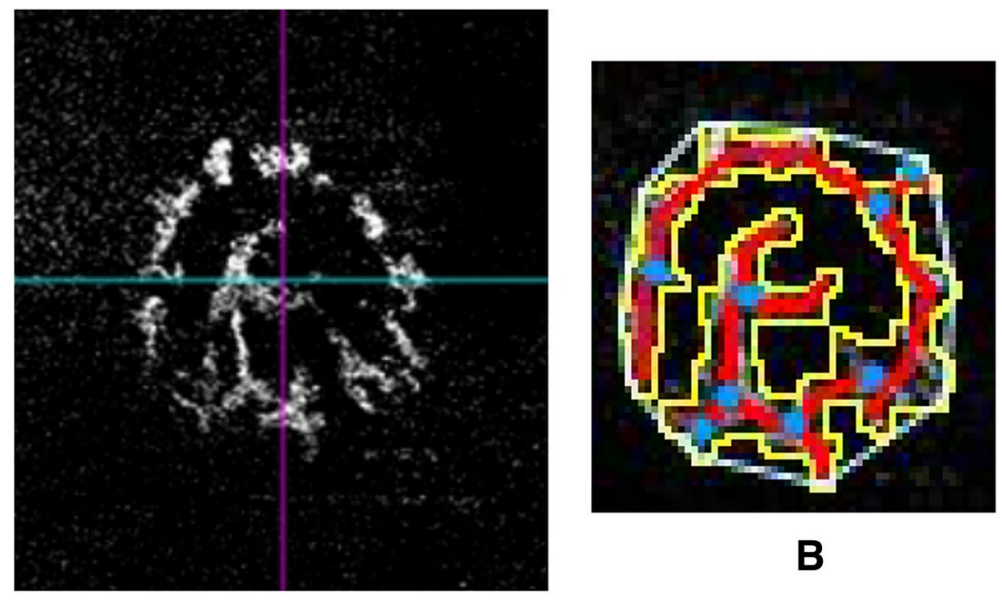

B

A

Figure 1. Analysis of OCTA parameters of the ONH at the age of 4 weeks using AngioTool software. (A) Retinal avascular layers segmentation mode was applied to the $\mathrm{ONH}$ and selected by the inbuilt software of OCTA and were subsequently imported to the AngioTool software. (B) The AngioTool software automatically measured the explant area (area within blue circles), vessel area (area within yellow circles), vascular density, total number of junctions (blue dots), junction density, total vessel length (red lines), average vessel length, and the total number of endpoints of vessels. OCTA optical coherence tomography angiography, ONH optic nerve head.

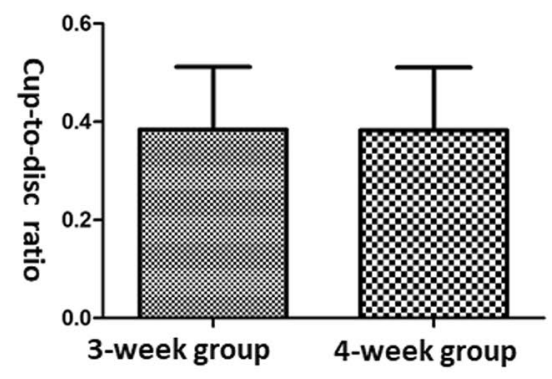

A

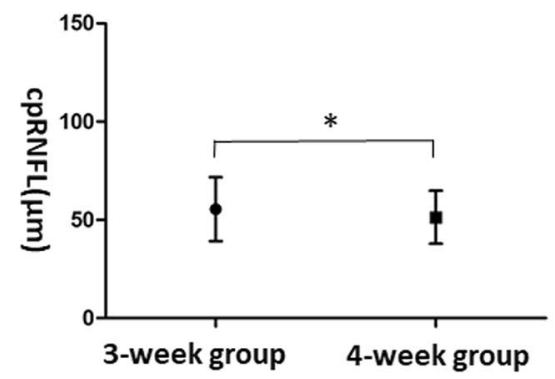

B

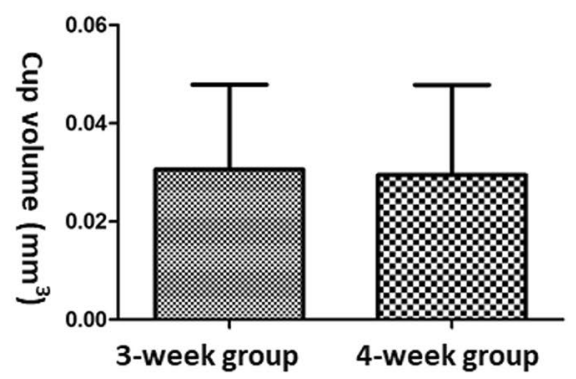

C

Figure 2. Morphological parameters of the optic nerve head in normal guinea pigs. (A) Cup-to-disc ratio: $0.38 \pm 0.128$ (3-week group) and $0.38 \pm 0.128$ (4-week group). (B) Thickness of circumpapillary retinal nerve fibre layer: $55.4 \pm 16.27 \mu \mathrm{m}$ (3-week group) and $51.3 \pm 13.45 \mu \mathrm{m}$ (4-week group). (C) Cup volume: $0.031 \pm 0.0173$ $\mathrm{mm}^{3}$ (3-week group) and $0.029 \pm 0.0184 \mathrm{~mm}^{3}$ (4-week group). ${ }^{*} \mathrm{p}<0.05$.

\section{Results}

General information. A total of 208 right eyes from 208 normal guinea pigs were included, which comprised 83 3-week-old (3-week group) and 125 4-week-old (4-week group) guinea pig eyes. The average AL, refractive error, and IOP were $7.68 \pm 0.21 \mathrm{~mm},+2.38 \pm 2.10 \mathrm{D}$, and $17.4 \pm 3.04 \mathrm{mmHg}$, respectively. No significant difference in AL was found between the 3-week and 4-week groups $(7.67 \pm 0.19 \mathrm{~mm}$ vs. $7.69 \pm 0.22 \mathrm{~mm}$, $\mathrm{t}=0.432, \mathrm{p}=0.666)$. The refractive error in the 3 -week group was significantly larger than that in the 4 -week group (+3.02 $\pm 1.27 \mathrm{D}$ vs. $+1.96 \pm 2.37 \mathrm{D}, \mathrm{t}=3.710, \mathrm{p}<0.001)$. The IOP in the 3 -week group was significantly lower than that in the 4 -week group $(16.7 \pm 3.19 \mathrm{mmHg}$ vs. $17.8 \pm 2.88 \mathrm{mmHg}, \mathrm{t}=2.483, \mathrm{p}=0.014)$.

Morphological parameters of the optic disc based on OCT. The cup-to-disc ratio, cpRNFL thickness, and cup volume of the 208 eyes were $0.38 \pm 0.13,53.0 \pm 14.74 \mu \mathrm{m}$, and $0.030 \pm 0.018 \mathrm{~mm}^{3}$, respectively. The cpRNFL thickness in the 3-week group was significantly larger than that in the 4-week group $(55.4 \pm 16.27 \mu \mathrm{m}$ vs. $51.3 \pm 13.45 \mu \mathrm{m}, \mathrm{t}=1.993, \mathrm{p}=0.048$ ). However, no significant differences were found in the cup-to-disc ratio and cup volume between the 3-week and 4-week groups (Fig. 2).

Blood perfusion in the ONH based on OCTA. The vessel area, vascular density, total number of junctions, total vessel length, total number of endpoints, and vascular diameter were $924.64 \pm 226.33$ pixels, 


\begin{tabular}{|l|l|l|l|l|l|l|l|}
\hline & $\mathbf{N}$ & Vessel area (pixels) & Vascular density (\%) & $\begin{array}{l}\text { Total number of } \\
\text { junctions }(\mathbf{N})\end{array}$ & $\begin{array}{l}\text { Total vessel length } \\
\text { (pixels) }\end{array}$ & $\begin{array}{l}\text { Total number of } \\
\text { endpoints (N) }\end{array}$ & $\begin{array}{l}\text { Vascular diameter } \\
\text { (pixels) }\end{array}$ \\
\hline 3-week group & 83 eyes & $920.63 \pm 227.994$ & $48.44 \pm 7.493$ & $6.6 \pm 3.39$ & $180.81 \pm 49.427$ & $7.6 \pm 2.88$ & $5.24 \pm 1.167$ \\
\hline 4-week group & 125 eyes & $927.31 \pm 226.097$ & $49.15 \pm 6.871$ & $7.0 \pm 3.64$ & $180.63 \pm 49.640$ & $8.2 \pm 3.38$ & $5.26 \pm 0.927$ \\
\hline & & $\mathrm{t}=0.208, \mathrm{p}=0.835$ & $\mathrm{t}=0.700, \mathrm{p}=0.485$ & $\mathrm{t}=0.897, \mathrm{p}=0.371$ & $\mathrm{t}=0.026, \mathrm{p}=0.979$ & $\mathrm{t}=1.394, \mathrm{p}=0.165$ & $\mathrm{t}=0.118, \mathrm{p}=0.906$ \\
\hline
\end{tabular}

Table 1. Blood flow parameters of the optic disc head based on OCTA.

$48.86 \pm 7.12 \%, 6.84 \pm 3.54,180.70 \pm 49.44$ pixels, $7.93 \pm 3.20$, and $5.25 \pm 1.03$ pixels, respectively. There were no significant differences in any of these parameters between the 3-week and 4-week groups (Table 1).

Factors influencing the vascular density and total vessel length of the ONH. The vascular density of ONH was positively correlated with vessel area $(\mathrm{r}=0.508, \mathrm{p}<0.001)$, total number of junctions $(\mathrm{r}=0.348$, $\mathrm{p}<0.001)$, total vessel length $(\mathrm{r}=0.265, \mathrm{p}<0.001)$, and vascular diameter $(\mathrm{r}=0.241, \mathrm{p}<0.001)$ and negatively correlated with the total number of endpoints $(\mathrm{r}=-0.460, \mathrm{p}<0.001)$. However, an age-adjusted linear regression analysis revealed that vascular density was positively associated with vessel area $(B=0.036 ; 95 \%$ confidence interval $[\mathrm{CI}] 0.029$ to $0.043 ; \mathrm{p}<0.001)$ and the total number of junctions $(\mathrm{B}=1.363 ; 95 \% \mathrm{CI} 1.096$ to 1.629 ; $\mathrm{p}<0.001)$ and negatively associated with the total vessel length $(B=-0.172 ; 95 \% C I-0.211$ to $-0.132 ; p<0.001)$, total number of endpoints $(B=-1.418 ; 95 \% \mathrm{CI}-1.576$ to $-1.260 ; \mathrm{p}<0.001)$, and vascular diameter $(\mathrm{B}=-0.647$; $95 \% \mathrm{CI}-1.490$ to $0.197 ; \mathrm{p}<0.001)$.

The total vessel length of ONH was positively correlated with vessel area $(r=0.870, p<0.001)$, vascular density $(0.265, \mathrm{p}<0.001)$, total number of junctions $(\mathrm{r}=0.853, \mathrm{p}<0.001)$, and total number of endpoints $(\mathrm{r}=0.257$, $\mathrm{p}<0.001)$. However, it was negatively associated with the vascular diameter of ONH $(\mathrm{r}=-0.469, \mathrm{p}<0.001)$. cpRNFL thickness $(r=0.110, p=0.113)$ and IOP $(r=0.076, p=0.273)$ did not show any relationships with total vessel length. However, an age-adjusted linear regression analysis revealed that the total vessel length was positively associated with vessel area $(B=0.166 ; 95 \%$ CI 0.155 to $0.177 ; p<0.001)$, total number of junctions $(B=4.413 ; 95 \% C I 3.632$ to $5.194 ; p<0.001)$, and cpRNFL thickness $(B=0.110 ; 95 \%$ CI 0.015 to $0.205 ; p=0.024)$ and negatively associated with vascular density $(\mathrm{B}=-1.622 ; 95 \% \mathrm{CI}-1.973$ to $-1.271 ; \mathrm{p}<0.001)$, total number of endpoints $(\mathrm{B}=-2.319 ; 95 \% \mathrm{CI}-3.002$ to $-1.636 ; \mathrm{p}<0.001)$, vascular diameter $(\mathrm{B}=-13.209 ; 95 \% \mathrm{CI}-14.920$ to $-11.497 ; \mathrm{p}<0.001)$, and IOP $(\mathrm{B}=-0.611 ; 95 \% \mathrm{CI}-1.108$ to $-0.114 ; \mathrm{p}=0.016)$.

\section{Discussion}

Guinea pigs have become increasingly popular models for studying human myopia ${ }^{3}$. Therefore, it is important to have data on the normal development of the eyes of guinea pigs. The main goals of this study were to set the normal ranges for both morphological and vascular parameters of the $\mathrm{ONH}$ in normal guinea pigs and analyse the factors influencing blood perfusion in the ONH.

Our results showed that no significant differences were found in the cup-to-disc ratio, and cup volume between the 3-week and 4-week groups. However, as no related analyses have been reported, we were unable to compare them to any corresponding reference values. RNFL thickness is an important clinical diagnostic measure for glaucoma ${ }^{11}$. In our study, the cpRNFL thickness of the 3-week group $(55.4 \pm 16.27 \mu \mathrm{m})$ was significantly larger than that of the 4 -week group $(51.3 \pm 13.45 \mu \mathrm{m})$. Jnawali et al. reported that the RNFL thickness of the 2.5-year-old pigmented guinea pigs was $59.2 \pm 4.5 \mu \mathrm{m}^{2}$, which was greater than our result.

OCTA and AngioTool software have been successfully used in clinicopathological studies with high accuracy and reproducibility. For example, Zimmermann et al. used AngioTool software to assess the performance of OCTA for imaging of the vascular network in the SVP and DVP and concluded that OCTA is a helpful imaging tool for non-invasive and in vivo imaging of the vascular plexus in mice. It may offer advantages over fluorescein angiography and confocal microscopy especially for imaging the deep vascular plexus ${ }^{10}$. Similarly, Liu et al. successfully extracted the retinal vasculature and quantified the retinal vessel branch points, vascular area, and vessel lengths using the AngioTool and concluded that simultaneous non-invasive analysis of the retinal vessels and neurons by OCTA and OCT may provide a novel approach for characterising retinal ischemia accompanied by neurovascular coupling ${ }^{12}$. Guinea pigs were used in the present study, and the structure and vasculature of $\mathrm{ONH}$, instead of the retina, were analysed. No previous study has reported on this, and our study focused on the vasculature of the $\mathrm{ONH}$. The vessel area, vascular density, total number of junctions, total vessel length, total number of endpoints, and vascular diameter were $924.64 \pm 226.33$ pixels, $48.86 \pm 7.12 \%, 6.84 \pm 3.54,180.70 \pm 49.44$ pixels, $7.93 \pm 3.20$, and $5.25 \pm 1.027$ pixels, respectively. It has been reported that the vascular density within the Bruch membrane opening of rhesus monkeys was $42.7 \%(10 \mathrm{mmHg}), 43.5 \%(20 \mathrm{mmHg})$, and $43.3 \%(30 \mathrm{mmHg})^{13}$.

Age-adjusted linear regression analysis revealed that the cpRNFL thickness is positively associated with total vessel length, and IOP is negatively associated with total vessel length. Manalastas et al. reported that the thicknesses of cpRNFL and the macular ganglion cell complex are more strongly associated with the vascular density of the ONH of glaucoma patients, glaucoma suspects, and healthy individuals ${ }^{14}$. Moreover, age-adjusted linear regression analysis revealed that vascular density is positively associated with vessel area and the total number of junctions and negatively associated with total vessel length, total number of endpoints, and vascular diameter.

Our results revealed that the $\mathrm{AL}$, refractive error, and IOP were $7.68 \pm 0.21 \mathrm{~mm},+2.38 \pm 2.10 \mathrm{D}$, and $17.4 \pm 3.04 \mathrm{mmHg}$, respectively. Jnawali et al. reported that the mean AL was $10.00 \pm 0.12 \mathrm{~mm}$ for guinea pigs aged 2.5 years $^{2}$. In addition, two previous studies on normal guinea pigs (age unknown) reported a mean IOP 
of $16.5 \pm 3.2 \mathrm{mmHg}$ for 100 normal guinea pigs ${ }^{15}$ and $18.27 \pm 4.55 \mathrm{mmHg}$ for 31 normal guinea pigs ${ }^{16}$. In our study, the IOP of the 3 -week group was significantly lower than that of the 4 -week group $(16.7 \pm 3.19 \mathrm{mmHg}$ vs. $17.8 \pm 2.88 \mathrm{mmHg}$ ). Similarly, Jnawali et al. reported that IOPs showed an early minimal increase that appeared to stabilise subsequently with age ${ }^{2}$.

Our study showed that the refractive error of the 3-week group was significantly greater than that of the 4-week group (+3.02 $\pm 1.27 \mathrm{D}$ vs. $+1.96 \pm 2.37 \mathrm{D})$. Jnawali et al. also reported that the young guinea pigs were hyperopic, with a mean spherical equivalent refraction of $+3.50 \pm 0.77 \mathrm{D}^{2}$.

No difference was found in AL between the 3-week and 4-week groups $(7.67 \pm 0.19 \mathrm{~mm}$ vs. $7.69 \pm 0.22 \mathrm{~mm})$. Similarly, Zhou et al. reported that the increase in AL was relatively rapid during the first week, followed by minimal changes during the next 3 weeks and rapid increments after 5 weeks ${ }^{8}$. The emmetropisation process in guinea pigs is mainly related to the increase in the vitreous chamber length, and the axial development of the vitreous chamber in guinea pigs appears to be associated with posterior scleral tissue growth ${ }^{8}$.

This study has limitations. First, we used the Cirrus OCT, which is designed for much larger human eyes, to assess the $\mathrm{ONH}$ of the eyes of the guinea pig. Second, we did not measure the refractive powers of the guinea pigs at birth. These limitations should be addressed in further research.

Nevertheless, this is the first report on the morphological and vascular characteristics of the ONH in normal guinea pigs based on in vivo OCT/OCTA imaging and quantification of ONH parameters. Hence, our results also corroborate the report that the maturation of guinea pig eyes continues in the early stages of life, and they may provide excellent models for myopic investigation.

\section{Data availability}

All data are included in the manuscript.

Received: 31 July 2021; Accepted: 4 January 2022

Published online: 18 January 2022

\section{References}

1. Xu, L., Wang, Y., Wang, S., Wang, Y. \& Jonas, J. B. High myopia and glaucoma susceptibility the Beijing Eye Study. Ophthalmology 114, 216-220 (2007)

2. Jnawali, A., Beach, K. M. \& Ostrin, L. A. In vivo imaging of the retina, choroid, and optic nerve head in guinea pigs. Curr. Eye Res. 43, 1006-1018 (2018).

3. Ostrin, L. A. \& Wildsoet, C. F. Optic nerve head and intraocular pressure in the guinea pig eye. Exp. Eye Res. 146, 7-16 (2016).

4. Cuenca, N. et al. Interpretation of OCT and OCTA images from a histological approach: Clinical and experimental implications. Prog. Retin. Eye Res. 77, 100828 (2020).

5. Hua, R. et al. Detection of preperimetric glaucoma using Bruch membrane opening, neural canal and posterior pole asymmetry analysis of optical coherence tomography. Sci. Rep. 6, 21743 (2016).

6. Zhang, S. et al. Changes in choroidal thickness and choroidal blood perfusion in guinea pig myopia. Investig. Ophthalmol. Vis. Sci. 60, 3074-3083. https://doi.org/10.1167/iovs.18-26397 (2019).

7. Meyer, J. H. et al. Optical coherence tomography angiography (OCT-A) in an animal model of laser-induced choroidal neovascularization. Exp. Eye Res. 184, 162-171 (2019).

8. Zhou, X. et al. Normal development of refractive state and ocular dimensions in guinea pigs. Vis. Res. 46, 2815-2823 (2006).

9. Zudaire, E., Gambardella, L., Kurcz, C. \& Vermeren, S. A computational tool for quantitative analysis of vascular networks. PLoS ONE 6, e27385 (2011).

10. Giannakaki-Zimmermann, H., Kokona, D., Wolf, S., Ebneter, A. \& Zinkernagel, M. S. Optical coherence tomography angiography in mice: Comparison with confocal scanning laser microscopy and fluorescein angiography. Transl. Vis. Sci. Technol. 5, 11 (2016).

11. Bowd, C., Weinreb, R. N. \& Zangwill, L. M. Evaluating the optic disc and retinal nerve fiber layer in glaucoma. I: Clinical examination and photographic methods. Semin. Ophthalmol. 15, 194-205 (2000).

12. Liu, W., Luisi, J., Liu, H., Motamedi, M. \& Zhang, W. OCT-Angiography for non-invasive monitoring of neuronal and vascular structure in mouse retina: Implication for characterization of retinal neurovascular coupling. EC Ophthalmol. 5, 89-98 (2017).

13. Patel, N., McAllister, F., Pardon, L. \& Harwerth, R. The effects of graded intraocular pressure challenge on the optic nerve head. Exp. Eye Res. 169, 79-90 (2018).

14. Manalastas, P. I. C. et al. The association between the macula and ONH optical coherence tomography angiography (OCT-A) vessel densities in glaucoma, glaucoma suspect, and healthy eyes. J. Glaucoma 27, 227-232 (2018).

15. Williams, D. \& Sullivan, A. Ocular disease in the guinea pig (Cavia porcellus): A survey of 1000 animals. Vet. Ophthalmol. 13, 54-62 (2010).

16. Coster, M. E., Stiles, J., Krohne, S. G. \& Raskin, R. E. Results of diagnostic ophthalmic testing in healthy guinea pigs. J. Am. Vet. Med. Assoc. 232, 18 (2008).

\section{Author contributions}

R.H., L.G., and J.T. made substantial contributions to conception and design, analysis and interpretation of data; R.H., and L.G. took part in drafting the article or revising it critically for important intellectual content; L.G., Y.T., S.C.C., and X.Z. performed the experiment and acquired data; all authors agreed to submit to the current journal; all authors gave final approval of the version to be published; all authors agree to be accountable for all aspects of the work.

\section{Funding}

This study was funded by the Beijing Bethune Charitable Foundation (No. AF-OG-03-1.1-03).

\section{Competing interests}

The authors declare no competing interests.

\section{Additional information}

Correspondence and requests for materials should be addressed to R.H. 
Reprints and permissions information is available at www.nature.com/reprints.

Publisher's note Springer Nature remains neutral with regard to jurisdictional claims in published maps and institutional affiliations.

(c) (i) Open Access This article is licensed under a Creative Commons Attribution 4.0 International License, which permits use, sharing, adaptation, distribution and reproduction in any medium or format, as long as you give appropriate credit to the original author(s) and the source, provide a link to the Creative Commons licence, and indicate if changes were made. The images or other third party material in this article are included in the article's Creative Commons licence, unless indicated otherwise in a credit line to the material. If material is not included in the article's Creative Commons licence and your intended use is not permitted by statutory regulation or exceeds the permitted use, you will need to obtain permission directly from the copyright holder. To view a copy of this licence, visit http://creativecommons.org/licenses/by/4.0/.

(c) The Author(s) 2022 